\title{
A Controlled Trial of Brief Intervention Versus Brief Advice for At-Risk Drinking Trauma Center Patients
}

\author{
Carl A. Soderstrom, MD, Carlo C. DiClemente, PhD, Patricia C. Dischinger, PhD, J. Richard Hebel, PhD, \\ David R. McDuff, MD, Kimberly Mitchell Auman, MS, and Joseph A. Kufera, MA
}

Background: Numerous reports document that preinjury alcohol use is associated with all modes of injury requiring treatment in a trauma center, with $25 \%$ to $50 \%$ or more of patients testing positive for alcohol at the time of admission. There is evidence that in trauma patients unaddressed alcohol use problems result in recurrent injury requiring readmission to a trauma center and/or death.

Methods: A randomized clinical trial was conducted to assess the effectiveness of two types of brief interventions to reduce drinking and the consequences of drinking. Trauma patients defined as atrisk alcohol users $(n=497)$ were ran- domized into two treatment options: a brief personalized motivational intervention (PMI), or brief information and advice (BIA). After a brief assessment, PMI subjects received a motivational session, feedback letter, and two postdischarge telephone contacts, whereas the BIA group received a brochure and one postdischarge telephone contact. Both groups were reassessed at 6 and 12 months postinjury.

Results: Both the PMI and BIA groups had statistically significant reductions in drinking, binge episodes, and consequences related to drinking that persisted from the 6- to the 12-month follow- up. However, although not statistically significant, for those classified as lowerlevel drinkers ( $\leq 1$ drink per day), there was a consistent pattern of maintaining reductions for the PMI group at 12 months compared with the BIA group.

Conclusion: Our results suggest that brief interventions (PMI and BIA) that link alcohol consumption with trauma injury and consequences of drinking can be effective in reducing drinking and consequences related to drinking in a significant portion of at-risk nondependent drinkers.
$\mathbf{N}$

umerous reports document that preinjury alcohol use is associated with all modes of injury requiring treatment in a trauma center, with $25 \%$ to $50 \%$ or more of patients ${ }^{1-5}$ testing positive for alcohol at the time of admission. Further, depending on whether screening test results or diagnostic criteria were used, $25 \%$ to $50 \%$ of trauma center patients have been identified to have an alcohol use problem. ${ }^{1,6,7}$ A prior study in the clinical site for the current study documented that $24 \%$ of admitted patients were alcohol dependent. $^{7}$

There is evidence that in trauma patients unaddressed alcohol use problems result in recurrent injury, requiring readmission to a trauma center and/or death. A 5-year longitudinal study ${ }^{8}$ of urban trauma center patients indicated that

Submitted for publication March 9, 2006.

Accepted for publication February 2, 2007

Copyright (C) 2007 by Lippincott Williams \& Wilkins, Inc.

From the National Center for Trauma and EMS (C.A.S., P.C.D., K.A., J.A.K.), the Departments of Epidemiology (J.R.H) and Psychiatry (D.R.M.), University of Maryland School of Medicine, Baltimore, MD; and the Department of Psychology (C.D.C), University of Maryland Baltimore County, Baltimore, MD.

Supported by the National Institute on Alcoholic Abuse and Alcoholism (grant 2 RO1 AA09050-04A2).

Presented orally at the Research Society on Alcoholism Meeting, June 27, 2005, Santa Barbara, California.

Address for reprints: Carl A. Soderstrom, MD, FACS, Maryland MVA, Medical Advisory Board, Glen Burnie, MD 21062; email: csoderstrom@umm. edu.

DOI: 10.1097/TA.0b013e31804bdb26 two-thirds of patients sustaining a recurrent injury had an indication of substance abuse (alcohol and/or drugs) at the time of the initial injury episode. Another study found that trauma center patients who were positive for possible alcoholism were over three times more likely to be readmitted for treatment of a second trauma episode compared with other patients. $^{9}$

The most compelling reason to conduct a trial of brief intervention for injured patients with alcohol use problems comes from a postdischarge mortality study from the trauma center that was the site for this project. ${ }^{10}$ In that study, over 27,000 patients were followed for a period of 1.5 to 14.5 years. In contrast to an overall $6.4 \%$ mortality rate from injury for the general population, $23 \%$ of trauma center patients died from a subsequent injury. Over one-third (35\%) of those who tested positive for alcohol or other drugs of abuse died as the result of a subsequent injury event after the initial trauma center admission (alcohol was the most common substance for which positive test results were obtained at the index injury admission). In contrast, $15 \%$ of trauma patients who tested negative died as the result of another injury episode.

A trauma center admission per se does not appear to result in decreases in postinjury hazardous drinking patterns. Using several measures of past-month hazardous drinking, Dunn and colleagues ${ }^{11}$ found that $41 \%$ of trauma center patients engaged in hazardous drinking before admission. Although there was an overall significant decrease in hazardous drinking among patients 1 month after injury, $55 \%$ of the 
patients reported hazardous drinking patterns 1 year after injury. In this same study, a positive alcohol test at the time of injury significantly predicted hazardous drinking 1 year after injury (odds ratio 9.18 with a $95 \%$ confidence interval of 2.51-33.56). In another study that provided no intervention for the control group of patients who evidenced alcohol use problems before admission, Gentilello and colleagues ${ }^{12}$ documented a dramatic decrease in consumption of drinks per week 6 months after discharge with a return to baseline drinking (or higher) at 1 year.

In 1981, Dr. Joseph J. Zuska, ${ }^{13}$ a surgeon interested in alcohol and its role in injury, noted, "The crisis that brings the alcoholic to the surgeon is an opportunity for intervention in a progressive, often fatal disease". Although the term "alcoholic" should be replaced with alcohol abuse and dependence in discussions of trauma patients, ${ }^{14}$ using techniques to intervene for alcohol use problems among injured patients has only recently been engaged. This has been in response to a growing body of evidence indicating that brief interventions in nonacute care settings can produce salutary results among problem drinking patients. ${ }^{15-17}$

In the study noted above, Gentilello and colleagues ${ }^{12}$ reported the results of a randomized clinical control trial of brief intervention for trauma center patients that produced a significant decrease in drinks consumed per week and a $47 \%$ reduction in injuries requiring emergency department care or trauma center admission at 1 year postintervention. A subsequent randomized control trial of a brief intervention in a population of injured drinkers treated in an emergency department also documented decreases in drinking at 1 year for both intervention and control subjects, but found significant decreases in alcohol-related consequences only among patients who had both the brief intervention and a second "booster" session postdischarge. ${ }^{18}$

In light of the above mentioned reports and others studies indicating the feasibility of performing interventions in trauma centers ${ }^{19}$ and emergency departments, ${ }^{20,21}$ a recommendation to "make screening, brief intervention, and referral to treatment routine practice in trauma centers" was promulgated after an intense 2.5-day dialogue among a group of trauma surgeons, emergency department physicians, substance abuse clinicians, health policy makers, and others in the spring of $2003 .^{22}$ Subsequently, the Committee on Trauma of the American College of Surgeons has adopted such a recommendation that both Level I and II trauma centers screen for alcohol use problems and that Level I centers offer intervention. ${ }^{23}$ With this information in hand, we present the results of a randomized clinical trial for a population of trauma center patients identified as at-risk drinkers. Two interventions, one based on the principles of motivational enhancement and the other a brief advice intervention, were compared for their impact on drinking outcomes and consequences of drinking.

\section{PATIENTS AND METHODS}

The study goal was to conduct a randomized clinical trial to compare the effectiveness of two types of interventionsbrief personalized motivational intervention (PMI) and brief information and advice (BIA) - to reduce drinking and consequences of drinking in a population of trauma center patients defined as at-risk alcohol users, such as abusive, problematic, and hazardous drinkers. These terms combined describe a group of "at-risk" drinkers who potentially can benefit from brief interventions. However, such interventions are not considered adequate/optimal to address the needs of patients with the most severe drinking problems. The project internally was named the "Delta Project" for the Greek letter often used to denote change in scientific nomenclature.

\section{Clinical Site}

The R Adams Cowley Shock Trauma Center (STC) at the University of Maryland Medical Center is the designated Level $\mathrm{I}^{24}$ regional adult trauma center for the most populated counties of central Maryland and a quadrant of Baltimore City. The patient population, which comes from rural, suburban, and urban settings, is representative of the aggregate of trauma patients treated in centers throughout the United States. ${ }^{25}$

\section{Delta Project}

The following describes the protocol for the Delta Project.

\section{Staffing}

Clinical Research Assistant (CRA). A full-time CRA screened and recruited eligible patients for the study. Study coverage was available 7 days a week.

Intervention Specialist (IS). During the course of the study, 14 clinical psychology doctoral graduate students at the University of Maryland, Baltimore County were trained to conduct intake interviews and deliver the PMI or BIA intervention options. Tape recordings of the interviews and interventions were made, with participant permission, and study supervisors reviewed a random $10 \%$ to $20 \%$ sample to monitor fidelity.

\section{Recruitment/Study Eligibility}

Initial Nursing Screen. Per clinical protocol, the Trauma Resuscitation Unit (TRU) nurse administered the "initial nursing screen" to all patients, which took 1 to 2 minutes to complete, and queried about alcohol use during the previous 24 hours, number of drinks per day, number of days per week of typical drinking, and the use of illicit drugs. This computerized report was reviewed daily by the CRA. Any of the following responses on this initial screen prompted a second screen by the CRA: (1) reported use of alcohol in the 24 hours before injury, (2) typical drinking of three or more drinks per day, (3) drinking four or more days in the past week, or (4) regular or current use of illicit drugs.

Eligibility Screen. The second screen, administered by the CRA, took 1 to 2 minutes to complete and was used to determine final subject eligibility. It consisted of the alco- 
hol CAGE questionnaire, ${ }^{26}$ three quantity and frequency questions from the Alcohol Use Disorders Identification Test (AUDIT), ${ }^{27,28}$ and a version of the CAGE to screen for drug use problems. After completion of the second screen, subjects meeting any of the following criteria were considered eligible for inclusion in the study: (1) any positive response to an item of the CAGE, or (2) drinking 2 or more times per week with total weekly drinking of 8 or more drinks for women and 15 or more drinks for men, or (3) drinking 2 to 4 times a month or less with typical daily consumption of 4 or more drinks for women and 5 or more drinks for men, or (4) drinking 6 or more drinks on one occasion, weekly, daily or almost daily. Blood alcohol testing was not a criterion for eligibility.

Additional Criteria. The following criteria also needed to be met for study eligibility: (1) being 18 years of age or greater, (2) having intact cognition, (3) having the ability to speak English, and (4) having a hospital length of stay (LOS) of 12 or more hours.

Exclusion Criteria. The following were considered exclusion criteria for the study: (1) unstable contact information, (2) legal issues (i.e., probation or parole), (3) a severe alcohol use problem considered inappropriate for a brief intervention, and (4) a severe drug use problem. Severe alcohol use was defined by at least one of the following: (a) the need for medical management for withdrawal and/or pain (secondary to cross tolerance to alcohol), (b) current enrollment in a formal alcohol treatment program (excluding self-help programs), or (c) meeting all of the following criteria: a CAGE score $>3$ for the past year; drinking four or more times per week; drinking six or more drinks on one occasion, daily or almost daily; and drinking four or more (female) or five or more (male) drinks. A severe drug use problem included (a) opiate and cocaine use, scoring $\geq 3$ on the drug CAGE and used 2 or more times a week in the past 90 days, (b) all other drug use, scoring $\geq 3$ more on the drug CAGE and participation in an active drug treatment program in the last year and used 2 or more times a week in the past 90 days.

Consent. The CRA conducted a brief (1- to 2-minute) assessment of cognition to determine ability to participate. Candidates had to demonstrate orientation to time, place, and person and good recall of recent and remote events. Informed consent was obtained by the CRA who then contacted the IS for the intake interview and intervention.

Note that screening by definition is a relatively inexact exercise. Our screening methodology was designed to include alcohol use problems that fall within the rubric of abusive/ hazardous/harmful alcohol use, with the intention of excluding patients with the most severe use problems. A review of the drinking patterns, including binge drinking episodes, of those recruited (see below and results) indicates that a small percentage of recruited subjects would be considered to have a severe alcohol use problem.

\section{Intake Interview}

The intake interview was administered by the IS in the acute care unit as soon as possible after consent and took about 25 minutes to complete. It consisted of the following:

1. Demographics (age, sex, marital status, driving record, prior injury).

2. Four risk-taking, sensation-seeking questions. ${ }^{29}$

3. SIP + 6: The 15-item Short Inventory of Problems (SIP), ${ }^{30}$ a brief version of the Drinking Inventory of Consequences (DrInC, 50 items), ${ }^{31}$ was used to assess lifetime and recent consequences of alcohol abuse. Six additional questions from the original DrInC about injury or drinking and driving were retained.

4. OASAS drinking history questions: alcohol consumption (quantity and frequency) was ascertained using questions from an instrument used in the New York Health Care Intervention Service Program Evaluation sponsored by the New York State Office of Alcoholism and Substance Abuse Services (OASAS) ${ }^{32}$ This seven-item instrument was modified to assess drinking patterns for the previous 3- and 1-month periods before injury.

5. Assessment of readiness and the stages of change to reduce alcohol consumption: a brief 12-item modified version of the University of Rhode Island Change Assessment Scale (URICA) ) $^{33-35}$ was used to measure attitudes and tasks related to precontemplation, contemplation, action, and maintenance stages of change ( 3 items per subscale). The short version demonstrated solid psychometric properties in terms of internal consistency and related as expected to other change process variables.

\section{Randomization of Subjects}

Upon completion of intake assessment, subjects were randomized into one of two intervention options: PMI or BIA. Randomization was achieved using a blind procedure whereby after the intake was completed the IS opened a sealed envelope, which indicated the subject's group assignment.

\section{Treatment Option 1}

PMI Protocol. The first component was a brief PMI counseling session. This session lasted about 15 to 20 minutes and took place as soon as feasible after the intake interview. Using motivational interviewing principles, the IS explored information obtained in the intake interview to help the subject evaluate his or her drinking patterns, assess motivations and barriers to change, explore motivation to modify drinking behaviors, and support any initiative to reduce or eliminate excessive drinking.

Feedback Letter. The second component of the PMI protocol was a personalized letter that summarized participant intake data and provided feedback based on responses to the questionnaires regarding problem dimensions, as well as stage of change, decisional balance, self efficacy, risk taking, 
stress, and social support, with some recommended actions. The letter was sent within 1 week of the subject's discharge. Postdischarge Telephone Contacts. The third component consisted of two telephone calls from the IS to the PMI subject. Several days after sending the feedback letter, the IS called to discuss it, including current motivation. Attempts were made to elicit a specific change plan regarding drinking behavior. This change plan was appropriate to stage status and designed to engage the subject in activities that would move them along the stages of change. The first call served as a reinforcement of the intervention delivered in the trauma center. The IS contacted PMI subjects again 4 weeks after trauma center discharge. During this call, the interventionist "checked in" on the change plan, reassessed motivation, discussed problem solving difficulties and barriers, supported self efficacy, and reinforced the need and motivation to modify drinking behavior. This second call concluded the PMI protocol.

\section{Treatment Option 2}

BIA Protocol. The IS informed the participants that their patterns of drinking put them at risk for future injury and gave advice to reduce their drinking. Each subject was given and asked to read a two-page handout that described causes of injury, the role of alcohol and injury, definition of a drink, types of drinkers, alcohol consumption in the United States, alcohol-related problems, and tips on how to reduce drinking or receive help.

Postdischarge Telephone Contact. For the second component of the BIA, protocol subjects were contacted by the IS 2 weeks after discharge. At this contact, the IS asked these subjects how they were doing, if they had had a chance to read the handout, and if they had any questions.

\section{6- and 12-Month Follow-up}

Telephone follow-up assessments for both the BIA and PMI groups occurred at 6 and 12 months postdischarge by an independent research assistant who also conducted collateral interviews with friends or family members of a randomly selected subsample of participants at each follow-up.

\section{Driving Citations}

Drivers' license data and driving citation data were obtained for the study participants from the Maryland Motor Vehicle Administration. Citations were classified as being alcohol related or nonalcohol related. For each participant identified as having a driver's license, we examined citations occurring in the year before and in the year after injury.

\section{Data Analysis}

Baseline analyses of subject characteristics involved comparisons between the PMI and BIA groups, as well as comparisons between subjects with complete and incomplete follow-up data. Differences were assessed using Pearson's $\chi^{2}$ statistic for categorical data and the Student's $t$ test for con- tinuous variables. A $p$ value of 0.05 or less was considered statistically significant.

Using the principle of intention to treat, longitudinal analyses were conducted by fitting mixed models to compare drinking outcomes for PMI participants relative to those in the BIA group. The trend of the outcome over time, from baseline to 6 and 12 months, was modeled by including, as independent variables, binary indicators for the treatment modality, the two follow-up time points, and treatment-bytime interactions. The statistical significance of a betweengroup difference in outcome over time was determined by testing the null hypothesis that the treatment and treatmentby-time interaction effects were simultaneously zero. Effectsize estimates and their associated confidence intervals were obtained for the 6- and 12-month outcomes, thus providing an indication of the longitudinal influence of the brief intervention.

Exploratory subgroup analyses were conducted to determine whether a particular type of drinker might derive some benefit from the brief intervention. Two baseline variables were used to classify the drinkers: number of drinks consumed during the previous 90 days and the number of binge drinking episodes occurring during the previous 90 days. For drinks in the past 90 days, lower (1-93 drinks), medium (94-225 drinks), and higher ( $\geq 226$ drinks) groups were defined based on tertile cut points. For number of binge drinking episodes in the past 90 days, lower ( $0-5$ episodes) and higher ( $\geq 13$ episodes) groups were based on a median cut point (upper and lower halves). (Note: there were no subjects who reported 6-12 binge episodes in 90 days.) The longitudinal analyses described above for all participants were repeated for the drinking subgroups.

Additional longitudinal analyses regarding the SIP score were conducted for all study participants. The SIP score is based on frequency responses to items describing events that are sometimes experienced by drinkers (e.g., "I have been unhappy because of my drinking"). Each response is scored as 0 (never), 1 (once or a few times), 2 (1-2 times per week), or 3 (daily or almost daily) before being summed and averaged across the 15 items to determine the mean score. Selfreport outcome data were also compared with reports for a small random sample of collaterals.

Because of the multiplicity of comparisons involved, the subgroup findings should be interpreted with caution. The $p$ values obtained from the longitudinal analyses were not used in a rigorous inferential context but rather as descriptive statistics for the purpose of identifying effects worthy of future investigation.

\section{RESULTS}

Study recruitment (September 2000 to November 2002) is graphically displayed in Figure 1. Of the 6,595 STC patients whose hospital stay was greater than 12 hours, 5,670 $(86 \%)$ were eligible for the first screening. Thirty-five percent $(\mathrm{n}=2,014)$ were positive on the first screen 


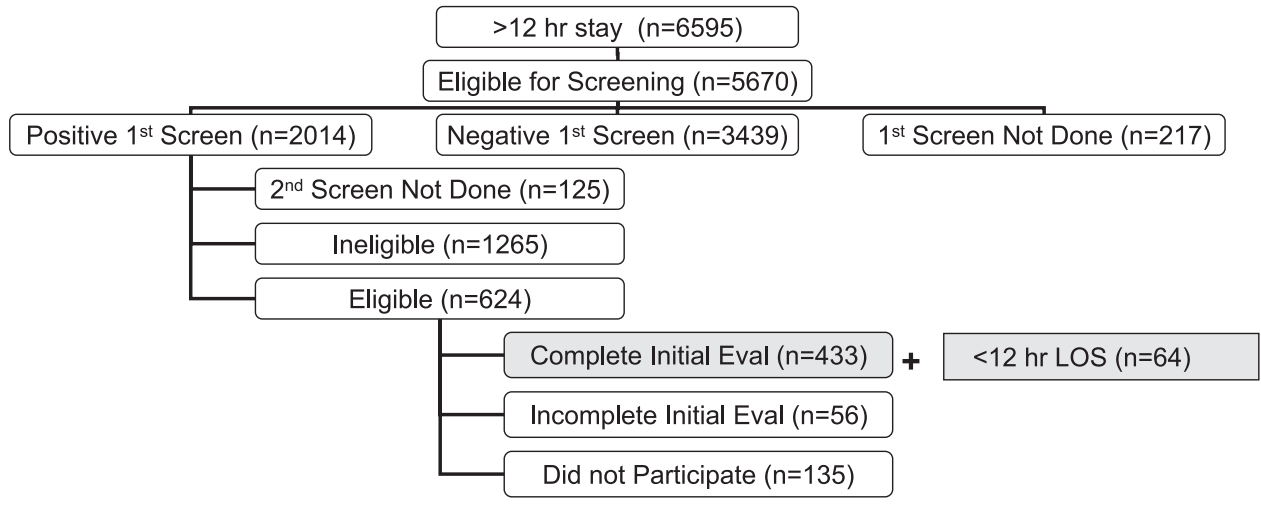

Fig. 1. Study recruitment.

Table 1 Baseline Demographic Characteristics:

Intervention vs. Controls $(\mathrm{N}=497)$

\begin{tabular}{lccc}
\hline & $\begin{array}{c}\text { Intervention } \\
(\mathrm{n}=250)\end{array}$ & $\begin{array}{c}\text { Control } \\
(\mathrm{n}=247)\end{array}$ & $p$ Value \\
\hline Male sex, \% & 84.8 & 85.4 & 0.85 \\
White race, \% & 63.1 & 62.6 & 0.93 \\
Blunt injury type, \% & 82.8 & 80.6 & 0.08 \\
Discharged to home, \% & 88.8 & 92.7 & 0.33 \\
Depressive symptoms, \% & 42.8 & 44.7 & 0.66 \\
Age, mean (SD) & $33.3(12.4)$ & $32.8(12.7)$ & 0.69 \\
$\quad$ No. drinks in 30 days, & $74.9(76.9)$ & $69.3(71.0)$ & 0.39 \\
$\quad$ mean (SD) & & & \\
No. drinks in 90 days, & $220.3(212.3)$ & $224.2(241.8)$ & 0.84 \\
$\quad$ mean (SD) & & & \\
No. binge episodes in & $7.4(9.0)$ & $7.5(8.7)$ & 0.96 \\
$\quad$ 30 days, mean (SD) & & & \\
No. binge episodes in & $20.7(24.8)$ & $22.1(26.2)$ & 0.51 \\
$\quad$ 90 days, mean (SD) & & & \\
\hline
\end{tabular}

indicating possible substance use-related issues. After administration of the second-level screening instrument, 624 patients remained eligible for the study. Of these, 433 (69\%) agreed to participate and completed the initial evaluation. To supplement recruitment efforts, an additional 64 subjects who were admitted and discharged from the STC within a 12-hour period were identified and able to complete the intake and interventions protocol. Thus, a total of 497 subjects completed the initial evaluation. Reasons for ineligibility include the following: not at-risk alcohol users (45\%), severe alcohol use (23\%), drug use (23\%), no contact information (3\%), and other reasons $(6 \%)$.

Upon completion of the intake measures, the 497 subjects were randomized into one of the two intervention options: 250 to the PMI group and 247 to the BIA group. There were no statistical differences between the two groups with respect to age, sex, race, mechanism of injury, discharge disposition, baseline depression index, or the number of drinks consumed during the previous 30- and 90-day periods (Table 1).

Of the 497 subjects completing the initial interview, $42 \%$ completed both the 6- and 12- month follow-up interviews. Overall, $71 \%(\mathrm{n}=354)$ of the study subjects completed at
Table 2 Baseline Demographic Characteristics: No Follow-up vs. Follow-up $(\mathrm{N}=497)$

\begin{tabular}{lccc}
\hline & $\begin{array}{c}\text { Follow-Up } \\
\text { No } \\
(\mathrm{n}=143)\end{array}$ & $\begin{array}{c}\text { Follow-Up } \\
\text { Yes } \\
(\mathrm{n}=354)\end{array}$ & $p$ Value \\
\hline $\begin{array}{lcc}\text { Intervention Study Arm, } \\
\mathrm{n} \text { [\%] }\end{array}$ & $71[49.7]$ & $179[50.6]$ & 0.85 \\
Male Sex & $128[89.5]$ & $295[83.3]$ & 0.08 \\
White Race & $76[53.2]$ & $235[66.8]$ & 0.004 \\
Blunt Injury type & $100[69.9]$ & $306[86.4]$ & 0.001 \\
Discharged to Home & $135[94.4]$ & $316[89.3]$ & 0.07 \\
$\begin{array}{l}\text { Depressive symptoms } \\
\text { Age, mean (SD) }\end{array}$ & $69[48.3]$ & $148[41.9]$ & 0.19 \\
No. drinks 30 days, mean & $31.4(12.2)$ & $33.8(12.6)$ & 0.05 \\
$\quad$ (SD) & $76.9(80.9)$ & $70.2(71.0)$ & 0.38 \\
No. drinks 90 days, mean & $234.5(253.3)$ & $217.3(216.0)$ & 0.48 \\
$\quad$ (SD) & & & \\
No. binge episodes 30 days, & $8.1(9.6)$ & $7.2(8.5)$ & 0.33 \\
$\quad$ mean (SD) & & & \\
No. binge episodes 90 days, & $22.1(27.2)$ & $21.1(24.8)$ & 0.71 \\
$\quad$ mean (SD) & & & \\
Injury Severity Score, median & $9(5-14)$ & $9(5-16)$ & 0.23 \\
$\quad$ (interquartile range) & $9(7-12)$ & $8(6-11)$ & 0.01 \\
$\begin{array}{l}\text { Perceived stress, median } \\
\text { (interquartile range) }\end{array}$ & & & \\
\hline
\end{tabular}

least one of the follow-up interviews (21\% 6-month only and $8 \%$ 12-month only).

Table 2 contains a comparison of baseline characteristics between those subjects who did and did not complete the 6and/or 12-month follow-up interviews. Study arm assignment did not differ between the followed and not followed groups. However, nonwhite subjects were lost to follow-up more often then white subjects $(p<0.004)$. Also subjects with penetrating injuries (i.e., gunshot, stab) were more likely to be lost to follow-up than were subjects with blunt injuries (i.e., motor vehicle crashes, falls). There were no significant differences in the quantity of drinks consumed at 30 and 90 days at baseline, although those who were followed were slightly older and tended to drink slightly less than did those who were not followed.

Longitudinal analyses of the number of drinks consumed during the previous 90 days by all study participants are 


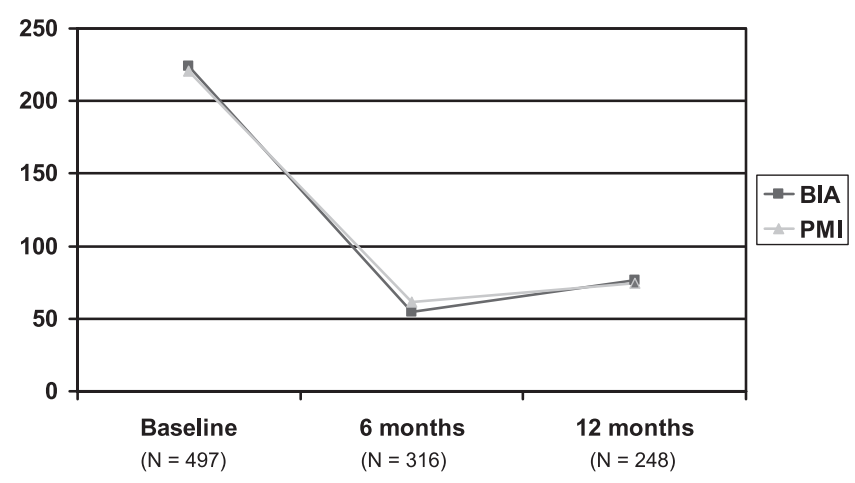

Fig. 2. Number of drinks within the past 90 days.

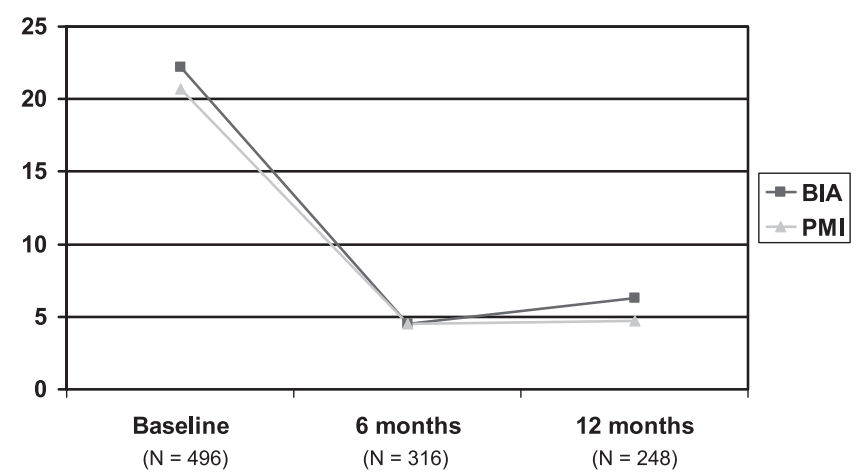

Fig. 3. Number of binges within the last 90 days.

presented in Figure 2. Both the PMI and the BIA groups are noted to have substantial and statistically significant decreases in drinking after baseline that persist from the 6- to the 12-month follow-up visit. There is no statistically significant difference in the two time trends $(p=0.97)$. Figure 3, indicates similar patterns for the number of binge drinking episodes occurring during the previous 90 days $(p=0.91)$.

Longitudinal analyses pertaining to a classification of the number of drinks consumed during the previous 90 days into the 3 subgroups (lower, medium, and higher) are shown in Figures 4 and 5. These time trends, for those whose baseline drinking was in either the medium (mean $=153$ drinks, $\mathrm{SD}=40$ drinks) or higher $($ mean $=477, \mathrm{SD}=224$ drinks $)$ categories, were remarkably similar to those seen for all participants. However, for those in the lower category ( 1 or fewer drinks per day during the previous 90 days, mean $=40$, $\mathrm{SD}=27$ drinks) with respect to either the number of drinks consumed or binge episodes occurring at baseline, there was a consistent pattern, although not statistically significant, of maintaining decreases in consumption $(p=0.32)$ and binge drinking episodes $(p=0.19)$ when compared with the other categories for the PMI group at 12 months versus the BIA group. This finding, although not to be taken as definitive, suggests that lower-level drinkers and binge drinkers were less convinced that drinking was a problem and were also least motivated to reduce their drinking.

Readiness to change was assessed using the URICA instrument. Analysis of variance (comparisons of readiness scores) differed significantly for groups based on numbers of drinks $(p<0.01)$ and numbers of binge drinking episodes $(p<0.05)$ in the past 90 days. Individuals in the lower drinks and binge groups had significantly lower readiness to change compared with the groups with the most drinks and binges.

Time trends analyses of the changes in drinking consequences (SIP scores) indicated that although the PMI and BIA group experienced substantial changes in consequences related to drinking after baseline at both 6 and 12 months, there was no statistically significant difference between the two time trends $(p=0.90)$. Contrary to the previous consumption subgroup analyses, however, the time trends for the lower number of drinks and number of binge drinking episodes categories, as well as the medium and higher level categories, were very similar to those seen for all participants and did not demonstrate group differences (data not shown).

Accuracy of self-reported consequences related to drinking as well as drinking patterns of study participants were evaluated by comparing responses of the participants with responses of the collaterals. There were no statistically significant differences between reports of consequences (e.g., driving after 3 or more drinks, getting into physical fights) at both the 6- and 12-month follow-up periods. Actually, participants reported significantly more drinking during the past 90 days at the 12-month interview, although $21 \%$ of the collateral respondents indicated that they did not know how much the subjects drank. Moreover, no differences were found by treatment condition.

Driver's license records were identified for $324(65 \%)$ of the study participants: 168 in PMI and 156 in BIA. There was no statistically significant difference in the rate of preinjury alcohol-related citations for either treatment option (PMI, $24 \%$ vs. BIA, $22 \% ; p=0.46$ ). However, when comparing postinjury alcohol-related citations among those with preinjury alcohol related citations (PMI, $\mathrm{n}=40$ and BIA, $\mathrm{n}=34$ ), the rate in the BIA group was double that of the PMI group $(14.7 \%$ vs. $7.5 \%, p=0.46)$.

\section{DISCUSSION}

To our knowledge, this is one of only a few large randomized trials comparing two types of interventions, a personalized motivational interview (PMI) and brief advice (BIA), for trauma patients, injured as the result of all types of trauma, who screened positive for an alcohol use problem. The study documents significant and salutary outcomes related to decreases in drinking and consequences of drinking, which can be achieved with personalized motivational intervention with feedback as well as with brief advice and information about drinking and injury. Although it is possible that these results could have been observed without any type of intervention, studies by Gentilello et al. ${ }^{12}$ and Dunn et al. ${ }^{11}$ do not support this view (see below). These overall salutary results for both types of interventions were more likely to be seen in drinkers in the medium to higher consumption groups in this study and those with more binge episodes. At-risk 
Lower Level Drinkers $(\mathrm{N}=165)$

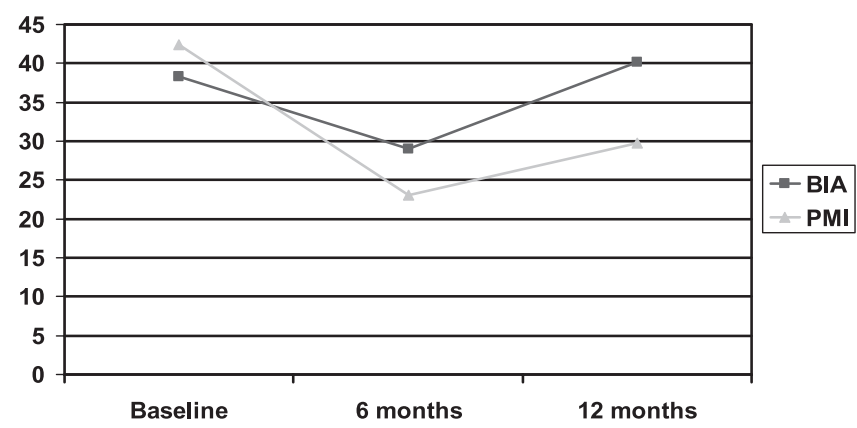

Medium Level Drinkers $(\mathrm{N}=167)$

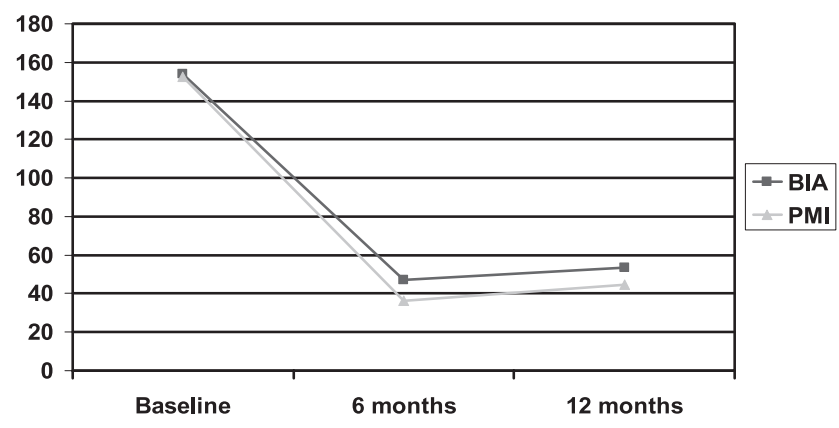

Higher Level Drinkers $(\mathrm{N}=164)$

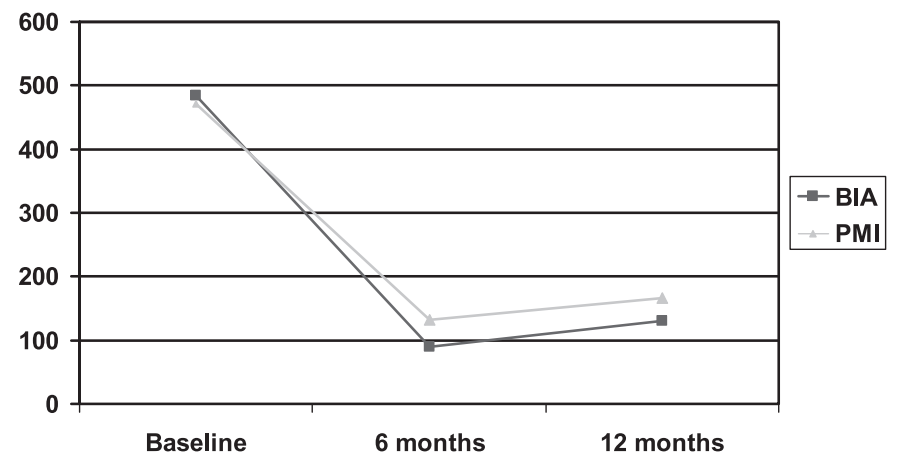

Fig. 4. Number of drinks within the last 90 days.

Lower Level Binge Drinkers

$$
(\mathrm{N}=229)
$$

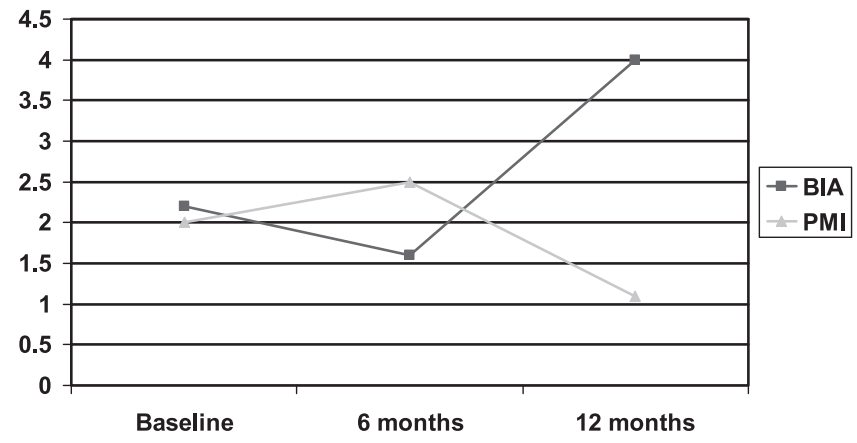

Fig. 5. Number of binges within the last 90 days.

drinkers with the lower levels of drinking benefited somewhat more from the personalized approach. PMI with the least problematic drinkers seemed to address their lack of motivation and disinterest in changing drinking better than those receiving brief advice, but these findings are suggestive since they were not statistically significant and lacked power to test for small differences. Drinkers with higher levels of drinking had more consequences and more motivation to change and were able to benefit from both PMI and BIA.

It is important to note limitations of this study when considering the results. Although we did not include a nointervention control group in this study, results demonstrate that providing interactive advice to trauma center patients
Higher Level Binge Drinkers

$$
(\mathrm{N}=267)
$$

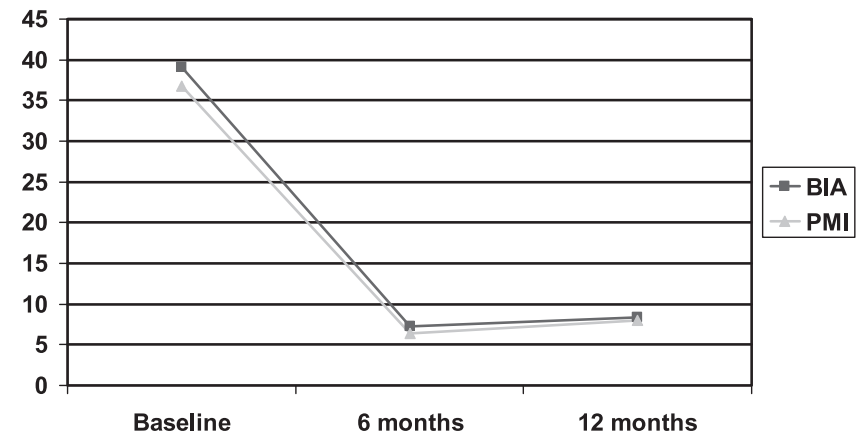

who screen positive for at-risk drinking results in decreases in drinking and consequences associated with such drinking. The dramatic reductions in numbers of drinks and binge drinking episodes were sustained at 12 months postintervention. Validity of drinking self reports were supported by reports of collaterals and preliminary analysis of posttreatment driving record data that were available on a subset of participants.

The $50 \%$ follow-up rate may adversely affect the results; however, the mixed model approach to longitudinal data analyses used early measurements available on each subject to adjust the follow-up results for missing observations. In addition, similar attrition rates for the PMI and BIA groups, 
as well as similar baseline attributes for those who did and did not complete follow-up, provide assurance that the between group comparison results will be relatively free of bias.

Our results are both similar to and differ from some other studies in trauma centers and emergency department settings. This Maryland study bears the closest resemblance to the study by Gentilello and colleagues ${ }^{12}$ that provided much of the impetus for both trauma surgery ${ }^{22,23}$ and emergency medicine ${ }^{36}$ communities to recommend use of screening and brief intervention in those acute care settings. In that study with a no-treatment control group, both control and intervention patients with moderate to high levels of hazardous drinking demonstrated significant reductions in the number of drinks consumed per week at 6 months. However, patients receiving the approximately 30-minute motivational intervention demonstrated greater sustained (12 months) reductions in drinking that differed significantly when compared with control subjects $(21.8 \pm 3.7$ drinks per week vs. $6.7 \pm 5.8$ drinks per week; $p=0.03$ ). That study also documented decreases in subsequent injury episodes requiring emergency department care or trauma center admission in favor of the intervention condition. In contrast, we documented overall similar significant decreases at 6 months in drinking (drinks and binge drinking in the past 90 days) for both our PMI and BIA subjects, which were sustained through 12 months. We also documented decreases in consequences for both PMI and BIA subjects at 6 and 12 months. Because the comparison group in this study differed dramatically in terms of amount (advice and information versus no intervention) and intensity (postdischarge contact), differences in study outcomes are reasonable. However, additional consideration of the differences in the methodologies of the two studies could be instructional as to what is needed in the trauma care setting to produce sustained changes in drinking.

The current study targeted patients with mild to moderate at-risk drinking problems. Those with evidence of alcohol dependence or significant "other drug" use were ineligible. In the Seattle study, enrolled subjects' alcohol use problems varied from mild to more severe and other drug use did not disqualify one for recruitment.

Unlike the current study, the Seattle study did not consent patients for enrollment into an alcohol intervention trial. Consenting to enrollment into an intervention study about alcohol use may have sensitized our patients to an awareness of their at-risk drinking and consequences of that drinking brought out by administration of intake measures. Further, by their agreement to participate in the current study it is possible that the subjects may have been contemplating decreasing (or even stopping) their alcohol use, whereas the Seattle study included more patients who were not ready to change. Hence, a possible explanation for the salutary results is that in the current study participants may have been more motivated and sensitized by the description of the study and administration of brief intake measures so that they needed less intervention. The Seattle population, which included a number of subjects with more severe drinking problems, may have benefited from administration of lengthy intake measures.

It is important to note that in the Seattle study, no benefit was noted at 1 year among patients with low scores on an alcohol screening questionnaire and those with very high scores, which suggested the possibility of dependence. Along the same lines, we found that although the lower-level drinkers and binge drinkers decreased their drinking at the 6-month interval, those who did not receive the PMI intervention returned to preinjury levels (or higher) at 12 months with a sharp upward trend in drinking 12 months after discharge among low-level bingers who received brief advice.

The current study may have had a "booster effect"18 for both the advice subjects and the PMI subjects who received a postdischarge phone contact 2 weeks after discharge to remind participants that they would be contacted at 6- and 12-month intervals and to request information if they had a change of address. Calls came from the interventionists who conducted their interview at the trauma center. Most subjects received one postdischarge call regardless of condition, so the anticipated dose difference in these calls did not materialize.

Although a host of studies have demonstrated the efficacy of brief interventions for patients with alcohol use problems in a variety of nonacute care settings, ${ }^{15-17,37,38}$ studies from acute care settings have yielded varying results. Longabaugh and colleagues ${ }^{18}$ documented decreased drinking 1 year after emergency department care among all the injured patients who were assessed to be hazardous or harmful drinkers and received any contact including a single session of brief intervention, brief intervention with a booster session, or the standard (no intervention) care. Further, only patients who received intervention booster sessions had significant decreases in alcohol-related consequences. In a study of nondependent patients injured in alcohol-related crashes, Sommers and colleagues ${ }^{19}$ found significant decreases in the incidence of binge drinking 12 months after acute care among those who received a brief intervention, those who received simple advice, and controls. Finally, D'Onofrio and colleagues $^{39}$ recently presented their results of a randomized control trial of brief intervention versus brief advice in a population of injured patients treated in an emergency department who were assessed to be hazardous/harmful drinkers. They noted significant decreases in harmful drinking 1 year after injury in both groups. These findings are quite similar to those obtained in our effort and in all these studies, which included an assessment of drinking and some additional contact that ranged from brief advice to little or no mention of drinking except in the consent and assessment protocols.

Relative to impaired driving convictions and intervention type, we found that in the year after intervention, the percentage of BIA subjects who had a conviction was twice that of PMI subjects ( $14.7 \%$ vs. $7.5 \%, p=0.46)$. Although these results were not statistically significant, they are consistent with other studies that demonstrate salutary results of inter- 
vention resulting in decreases in drinking and driving ${ }^{40}$ and impaired driving arrests. ${ }^{12}$

The important question posed by the current study and the studies discussed above $\mathrm{e}^{18,19,39}$ is, do interventions work? To answer that question, one must ask what constitutes an intervention. Previous, and indeed current, practice in many trauma centers is not to raise the issue of alcohol use. If raised, it is often in the form of a judgmental reprimand indicating that continued bad behavior (excessive drinking) will probably result in additional (justly deserved is implied) injury episodes. Simply raising the issue in a nonjudgmental empathic manner ${ }^{41}$ is a paradigm shift that links a consequence of drinking (injury) to at-risk drinking. In our study, the control group subjects were consented, completed intake/ assessment measures, advised about drinking and reinjury, and received a pamphlet and a follow-up telephone call. Hence, among all of these subjects who were hospitalized in a trauma center, some nonjudgmental attention was paid to their drinking and consequences of their drinking. This appears to have been enough to bring about changes in this group of at-risk alcohol-using patients.

Again, it is important to note that we attempted to exclude patients with the most severe drinking problems. Examination of the number of drinks and binge drinking episodes data for the recruited indicates that whereas a small percentage of patients with severe problems were included, the vast majority of recruited subjects could be characterized as at-risk, hazardous, problematic drinkers. Problem drinkers of the type recruited for study may have more self-regulating control of their drinking behavior. However, self-regulatory control is not the complete answer because those drinkers in our study with the lower levels of drinking, in terms of both number of drinks and binges at baseline, demonstrated greater benefit from the more personalized intervention at the 12-month follow-up. Motivation to change is also important because these lower-level drinkers had more absolute selfregulation of drinking but were probably the least motivated to change drinking patterns of the different drinking groups and seemed to be better candidates for the more extensive, personalized brief intervention.

Discussing the Gentilello et al. study, ${ }^{12}$ Dr. David Hoyt, then Chair of the American College of Surgeons' Committee on Trauma, said, "I would have to say that I was really skeptical regarding the value of this kind of intervention. It seems so relatively small in the context of everything else that is going on with these patients. I would also have to admit I have been wrong. It seems to work". Our study results suggest that for some patients a relatively small intervention of brief information and advice, which raises consciousness of alcohol misuse and its consequences (particularly injury) at two or more points of contacts (i.e., intake measures, brief advice handout, and postdischarge phone calls) may facilitate salutary changes. This is probably more likely to occur in patients with mild to moderate alcohol-use problems.
The acute care studies involving injured patients discussed above lend support to this hypothesis. Indeed, they lend support to Moyer and colleagues, ${ }^{38}$ observations in their meta-analysis of brief interventions for alcohol problems that the best results were obtained in groups of patients without severe alcohol problems who were not seeking treatment, such as the trauma patients involved in this study. Relative to these patients they noted, "the linkage of drinking to health problems, coupled with advice provided by a physician or nurse, may account (at least partially) for the positive effects of brief interventions in nontreatmentseeking populations".

The findings of this study support recommendations that screening and brief intervention become part of standard trauma center care. Our results indicate that, for a cadre of patients with mild to moderate alcohol-use problems, brief advice or formal brief intervention interviews are beneficial in reducing drinking and consequences of drinking.

\section{ACKNOWLEDGMENTS}

This study could not have been accomplished without the assistance of the following individuals: Mary Woodman, BS, and Paula Moylan, BS from National Study Center for Trauma and EMS, University of Maryland School of Medicine; Lori Bellino, PhD; Angela Marinilli, PhD; Danielle Black, MS; Judy Dedeyn, MS; Janine Delahanty, PhD; Amanda Gmyrek, MS; Sharon Hoover, MS; Amy Malkin, MS; Tara Neavins, MS; Melissa Nidecker, MS; Manu Singh, MS; Elise Vestal, MS; Catharine Weiss, MS; Shannon Whyte, MS; and Jamie Winters, MS; from the Department of Psychology, University of Maryland Baltimore County; Catonsville, MD; Christopher Welsh, MD; Eric Weintraub, MD; Mary Hirsch, RN; Lauren Broyles, RN; Anna Blasetti, BS; from the Department of Psychiatry, University of Maryland Medical Center; Baltimore, MD.

\section{REFERENCES}

1. Rivara FP, Jurkovich GJ, Gurney JG, et al. The magnitude of acute and chronic alcohol abuse in trauma patients. Arch Surg. 1993; 128:907-913.

2. Treno AJ, Cooper K, Roeper P. Estimating alcohol involvement in trauma patients: search for a surrogate. Alcohol Clin Exp Res. 1994; 18:1306-1311.

3. Cornwell EE, Belzberg H, Velmahos G, et al. The prevalence and effect of alcohol and drug abuse on cohort-matched critically injured patients. Am Surg. 1998;64:461-465.

4. Soderstrom CA, Dischinger PC, Kerns TJ, et al. Epidemic increases in cocaine and opiate use by trauma center patients: documentation with a large clinical toxicology database. J Trauma. 2001;51:557-564.

5. Blondell RD, Dodds HN, Looney SW, et al. Toxicology screening results: injury associations among hospitalized trauma patients. J Trauma. 2005;58:561-570.

6. Maio RF, Waller PF, Blow FC, et al. Alcohol abuse/dependence in motor vehicle crash victims presenting to the emergency department. Acad Emerg Med. 1997;4:256-262.

7. Soderstrom CA, Smith GS, Dischinger PC, et al. Psychoactive substance use disorders among seriously injured trauma center patients diagnosed using a standardized interview. JAMA. 1997; 277:1769-1774.

8. Sims DW, Bivins BA, Obeid FN, et al. Urban trauma: a chronic recurrent disease. J Trauma. 1989;29:940-947.

9. Rivara FP, Koepsell TD, Jurkovich GJ, et al. The effects of alcohol abuse on readmission for trauma. JAMA. 1993;270:1962-1964. 
10. Dischinger PC, Mitchell KA, Kufera JA, et al. A longitudinal study of former trauma center patients: the association between toxicology status and subsequent injury mortality. J Trauma. 2001;51:555-564.

11. Dunn C, Zatzick D, Russo J, et al. Hazardous drinking by trauma patients during the year after injury. J Trauma. 2003;54:707-712.

12. Gentilello LM, Rivara FP, Donovan DM, et al. Alcohol interventions in a trauma center as a means of reducing the risk of injury recurrence. Ann Surg. 1999;230:473-483.

13. Zuska, JJ. Wounds without cause. Bull Am Coll Surg. 1981;66:5-10.

14. Soderstrom CA. Session 2: substance abuse interventions-setting the stage for discussion. J Trauma. 2005;59:S77-S79.

15. Bien TH, Miller WR, Tonigan JS. Brief interventions for alcohol problems: a review. Addiction. 1993;88:315-336.

16. Wilk AI, Jensen NM, Havighurst TC. Meta-analysis of randomized clinical trials addressing brief interventions in heavy alcohol drinkers. J Gen Intern Med. 1997;12:274-283.

17. Dinh-Zarr T, Diguiseppi C, Heitman E, et al. Preventing injuries through interventions for problem drinking: a systematic review of randomized controlled trials. Alcohol. 1999;34:609-621.

18. Longabaugh R, Woolard RF, Nirenberg TD, et al. Evaluating the effects of a brief motivational intervention for injured drinkers in the emergency department. J Stud Alcohol. 2001;62:806-816.

19. Sommers MS, Dyehouse JM, Howe SR, et al. Effectiveness of brief interventions following alcohol-related vehicular injury: a randomized controlled trial. J Trauma. 2006;61:523-533.

20. D'Onofrio G, Degutis L. Preventive care in the emergency department: screening and brief intervention for alcohol problems in the emergency department: a systemic review. Acad Emerg Med. 2002;9:627-638.

21. Hungerford DW, Pollock DA, Todd KH. Acceptability of emergency department-based screening and brief intervention for alcohol problems. Acad Emerg Med. 2000;7:1383-1392.

22. Hungerford DW. Recommendations for trauma centers to improve screening, brief intervention, and referral to treatment for substance use disorders. J Trauma. 2005;59:S37-S42.

23. Committee on Trauma, American College of Surgeons. Resources for Optimal Care of the Injured Patient: 2006. Chicago, IL; American College of Surgeons, 2006.

24. Committee on Trauma, American College of Surgeons. Resources for Optimal Care of the Injured Patient: 1999. Chicago, IL; American College of Surgeons, 1999.

25. American College of Surgeons: National Trauma Data Bank: Annual Report 2005. Available at http://www.facs.org/trauma/ntdb/ ntdbannualreport2005.pdf. Accessed September 30, 2005.

26. Ewing JA. Detecting alcoholism: the CAGE questionnaire. JAMA. 1984;252:1905-1907.

27. Saunders JB, Aasland OG, Babor TF, et al. Development of the Alcohol Use Disorders Identification Test (AUDIT): WHO collaborative project on early detection of persons with harmful alcohol consumption, II. Addiction. 1993;88:791-804.

28. Babor TF, de la Fuente JR, Saunders J, Grant M: AUDIT: The Alcohol Use Disorders Identification Test: Guidelines for Use in Primary Health Care. Geneva, Switzerland: World Health Organization, 1992.

29. Soderstrom CA, Ballesteros MF, Dischinger PC, et al. Alcohol/drug abuse, driving convictions, and risk-taking dispositions among trauma center patients. Accid Anal Prev. 2001;33:771-782.

30. Center on Alcoholism, Substance Abuse and Addictions. Short inventory of Problems. Albuquerque, NM; Center on Alcoholism, Substance Abuse and Addictions; 1994.

31. Miller WR, Tonigan JS, Longabaugh R. The Drinker Inventory of Consequences (DrInC): An Instrument for Assessing Adverse Consequences of Alcohol Abuse. Test Manual. Project MATCH Monograph Series. Vol. 4. NIH Pub. No. 95-3911. Washington, DC: U.S. Government Printing Office, 1995.
32. Welte J, Longabaugh R, Clifford P, et al. The New York State Health Care Intervention Service Program Evaluation: Technical Summary of the Outcome Evaluation. Albany, NY: New York State Office of Alcoholism and Substance Abuse Services, 1996.

33. DiClemente CC, Schlundt D, Gemell L. Readiness and Stages of Change in Addiction Treatment. Am J Addict. 2004;13:103-119.

34. DiClemente CC, Hughes SO. Stages of change profiles in alcoholism treatment. J Subst Abuse. 1990;2:217-235.

35. Project MATCH Research Group. Matching alcoholism treatment to client heterogeneity: Project MATCH post-treatment outcomes. J Stud Alcohol. 1997;58:7-29.

36. Runge JW, Hargarten S, Velianoff G, et al. Developing Best Practices of Emergency Care for the Alcohol-Impaired Patient: Recommendations from the National Conference. Washington, DC: National Highway Traffic Safety Administration; 2001.

37. Dunn C, Deroo L, Rivara FP. The use of brief interventions adapted from motivational interviewing across behavioral domains: a systematic review. Addiction. 2001;96:1725-1742.

38. Moyers A, Finney JW, Swearingen CE, et al. Brief interventions for alcohol problems: a meta-analytic review of controlled investigations in treatment-seeking and non-treatment- seeking populations. Addiction. 2002;97:279-292.

39. D'Onofrio G, Degutis LC, Fiellen DA, et al. Emergency practitioner performed brief intervention for harmful and hazardous drinkers in the emergency department. Alcohol Clin Exp Res. 2005;28:179A.

40. Monti PM, Colby SM, Barnett NP, et al. Brief intervention for harm reduction with alcohol-positive older adolescents in a hospital emergency department. J Consult Clin Psychol. 1999;67:989-994.

41. Dunn C, Ostafin B. Brief interventions for hospitalized trauma patients. J Trauma. 2005;59;S88-S93.

\section{EDITORIAL COMMENT}

Injuries are the leading cause of mortality for problem drinkers, and cause more deaths in these patients than cirrhosis, pancreatitis, hepatitis, and all other alcohol-related medical conditions combined. ${ }^{1}$ Alcohol problems are to the trauma surgeon what smoking is to the pulmonologist, and what hypertension is to the cardiologist. Therefore, it is gratifying to see that this study by Soderstrom and colleagues supports the provision of alcohol screening and brief intervention $(\mathrm{SBI})$ in trauma centers.

The study compared two different "doses" of intervention. Patients were randomized to either a 20-minute motivational intervention, or to receive only 5 minutes of brief advice. Surprisingly, both groups had similar reductions in alcohol intake. The results can be interpreted in several ways. One would be that SBI is not warranted, as it performed no better than simple advice. This raises the question of whether either intervention had any beneficial effect. It is difficult to be certain without a control group. It may be that an alcoholrelated injury requiring trauma center admission causes patients to reduce their drinking, even without an intervention. The authors make a compelling argument that this is not the case. The high rate of trauma recidivism in problem drinkers, and their high mortality after discharge, strongly suggest that a trauma center admission alone is not a cure for a drinking problem.

An alternative interpretation, one provided by the authors, is that both interventions worked equally well. They suggest that admission to a trauma center increases respon- 
siveness to an intervention to the extent that only rudimentary intervention services are needed. One should be careful of this interpretation. Although the brief advice group reduced their drinking, the therapeutic effect may not have been a result of the advice alone. The screening and baseline assessment process included a 25 minute interview with an intervention specialist who extensively reviewed the patient's drinking history and drinking-related consequences experienced during their lifetime, questioned them about their drinking and driving, risky behaviors, and usual quantity of alcohol consumed, and assessed their motivation to change their drinking habits. The interventionist then provided them with information about how their drinking put them at risk for future injury, and gave them advice to cut down. In other words, even the five minute brief advice group spent at least 30 minutes with an intervention specialist.

This study also does not provide clear evidence of the minimum dose of intervention required in trauma centers because the entry criteria excluded patients with a serious drinking problem. Forty-five percent of those who screened positive for an alcohol use disorder were excluded because their problem was too severe, or because they also had a drug problem. This resulted in a study group with relatively mild drinking problems. The average alcohol intake of one-third of the patients was less than 1 drink per day, or only 40 drinks total in the 90-day period before enrollment. The lack of added effect of formal SBI over simple advice may in part be explained by the fact that for many patients, not much change occurred because not that much change was needed.
Overall, the entire study population average consumption at study entry was just over 70 drinks per month. This is much less alcohol use than is seen in the typical trauma patient with an alcohol use disorder, many of whom may not respond to simple advice, and might optimally receive some form of assistance that is even more extensive than SBI. It would be unfortunate if the results of this study are not put into perspective, and are used to minimize the extent of alcohol counseling services that should be available to injured patients.

Nevertheless, this is a study with a positive result, and with an overall message of optimism. Many health care providers are nihilistic when it comes to patients with alcohol problems, and think that change is unlikely, or impossible. ${ }^{2}$ This study conclusively demonstrates that many problem drinking patients admitted to a trauma center can and do get well, especially when offered a helping hand.

\section{Larry M. Gentilello, MD}

Division of Burns, Trauma, and Critical Care

University of Texas Southwestern Medical Center

Parkland Memorial Hospital

Dallas, Texas

\section{REFERENCES}

1. Centers for Disease Control. Morbidity and Mortality Weekly Report. 2004;53:866-870.

2. Danielsson PE, Rivara FP, Gentilello LM, et. al. Reasons why trauma surgeons fail to screen for alcohol problems. Arch Surgery. 1999;134:564-568. 